\title{
Plasma levels of glucagon but not GLP-1 are elevated in response to inflammation in humans
}

\author{
Justyna Modrzynska ${ }^{1, *}$, Christine F Klein ${ }^{2, *}$, Kasper Iversen ${ }^{3}$, Henning Bundgaard ${ }^{4}$, Bolette Hartmann ${ }^{1,5}$, \\ Maike Mose ${ }^{6}$, Nikolaj Rittig7, Niels Møller ${ }^{8}$, Jens J Holst ${ }^{1,5}$ and Nicolai J Wewer Albrechtsen 1,9,10 \\ ${ }^{1}$ Department of Biomedical Sciences, Faculty of Health and Medical Sciences, University of Copenhagen, Copenhagen, Denmark \\ ${ }^{2}$ Department of Cardiology, Herlev Gentofte Hospital, Herlev, Denmark \\ ${ }^{3}$ Department of Clinical Medicine, Herlev Gentofte Hospital, Herlev, Denmark \\ ${ }^{4}$ Department of Cardiology, Rigshospitalet, University of Copenhagen, Copenhagen, Denmark \\ ${ }^{5}$ Novo Nordisk Foundation Center for Basic Metabolic Research, Faculty of Health and Medical Sciences, University of Copenhagen, \\ Copenhagen, Denmark \\ ${ }^{6}$ Department of Endocrinology and Internal Medicine, Aarhus University Hospital, Aarhus, Denmark \\ ${ }^{7}$ Steno Diabetes Center Aarhus, Aarhus University Hospital, Aarhus, Denmark \\ ${ }^{8}$ Department of Clinical Medicine, Aarhus University Hospital, Aarhus, Denmark \\ ${ }^{9}$ Novo Nordisk Foundation Center for Protein Research, Faculty of Health and Medical Sciences, University of Copenhagen, Copenhagen, Denmark \\ ${ }^{10}$ Department of Clinical Biochemistry, Rigshospitalet, University of Copenhagen, Copenhagen, Denmark
}

Correspondence should be addressed to N J Wewer Albrechtsen: nicolai.albrechtsen@sund.ku.dk

*(J Modrzynska and C F Klein contributed equally to this work)

\section{Abstract}

Objective: Glucagon and glucagon-like peptide-1 (GLP-1) originate from the common precursor, proglucagon, and their plasma concentrations have been reported to be increased during inflammatory conditions. Increased blood glucose levels are frequently observed in septic patients, and therefore we hypothesized that glucagon, but not GLP-1, is increased in individuals with inflammation.

Design: Prospective longitudinal cohort study.

Materials and methods: We measured glucagon and GLP-1 in plasma sampled consecutively in three cohorts consisting of patients with infective endocarditis $(n=16)$, urosepsis $(n=28)$ and post-operative inflammation following percutaneous aortic valve implantation or thoracic endovascular aortic repair $(n=5)$. Correlations between C-reactive protein (CRP), a marker of systemic inflammation, and glucagon and GLP-1 concentrations were investigated. Additionally, glucagon and GLP-1 concentrations were measured after a bolus infusion of lipopolysaccharide (LPS, $1 \mathrm{ng} / \mathrm{kg}$ ) in nine healthy young males.

Results: Glucagon and CRP were positively and significantly correlated $(r=0.27$; $P=0.0003$ ), whereas no significant association between GLP-1 and CRP was found $(r=0.08, P=0.30)$. LPS infusion resulted in acute systemic inflammation reflected by increased temperature, pulse, tumor necrosis factor- $\alpha$ (TNF $\alpha)$, interleukin- 6 (IL-6) and concomitantly increased concentrations of glucagon $(P<0.05)$ but not GLP-1. Conclusions: Systemic inflammation caused by bacterial infections or developed as a noninfected condition is associated with increased plasma concentration of glucagon, but not GLP-1. Hyperglucagonemia may contribute to the impaired glucose control in patients with systemic inflammatory diseases.

\section{Key Words}

- bacteremia

- ELISA

- glucose

- hyperglycemia

- immunoassay

- infection

- inflammation https://ec.bioscientifica.com

https://doi.org/10.1530/EC-20-0590 (c) 2021 The authors Published by Bioscientifica Ltd

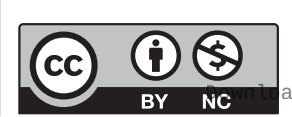

This work is licensed under a Creative Commons Attribution-NonCommercial 4.0 International License. ded from Bioscientifica.com at 04/26/2023 05:14:09AM
Endocrine Connections (2021) 10, 205-213 


\section{Introduction}

Glucagon and glucagon-like peptide-1 (GLP-1) are processed from the same precursor, proglucagon (Fig. 1), and have opposite effects on glucose homeostasis $(1,2,3)$. In the intestine, proglucagon is cleaved by prohormone convertase 1 (PC1) resulting in the formation of GLP-1, whereas in the pancreas proglucagon is processed by prohormone convertase 2 (PC2) leading to the formation of glucagon $(4,5)$ (Fig. 1).

Glucagon stimulates hepatic glucose production and thereby increases blood glucose levels, whereas GLP-1 potentiates glucose-induced insulin secretion from pancreatic $\beta$-cells and inhibits glucagon secretion, collectively resulting in a glucose lowering effect. GLP-1 receptor agonists (GLP-1RA) are therefore used as a treatment of type 2 diabetes (T2D) (6). In addition to the glycemic effects, it has been suggested that GLP-1 exhibits cardio- and neuro-protection, together with inhibition of inflammation and apoptosis (6).

An excessive inflammatory response can cause severe organ dysfunction (7). Increased blood glucose levels are frequently observed in septic patients, independent of pre-existing disturbances in glucose metabolism $(8,9)$, and hyperglycemia is significantly associated with their increased mortality (10). Some studies have reported that glucagon and GLP-1 concentrations are elevated during trauma, inflammation and or sepsis $(11,12,13,14,15)$. However, sequence homology between glucagon and GLP-1, close resemblance to other circulating proglucagonderived peptides, and the fact that they circulate in low (picomolar) concentrations, make accurate measurements of these hormones difficult $(16,17,18,19)$.

Elevated blood glucose levels are frequently observed in septic patients, and therefore we hypothesized that

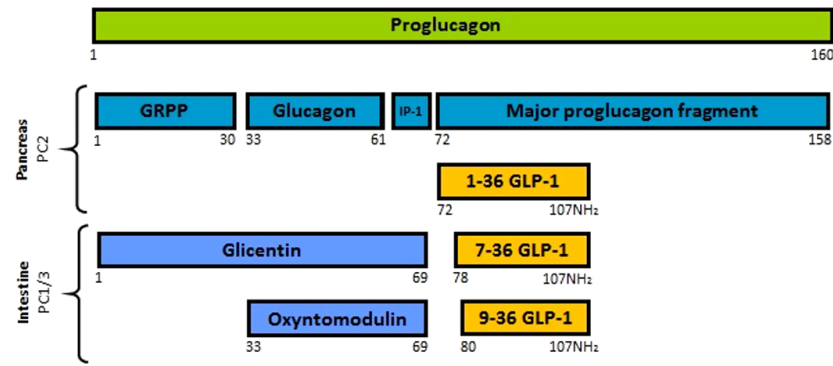

\section{Figure 1}

Overview of proglucagon molecular processing. Formation of intact active GLP-1 (7-36NH2) is mediated by prohormone convertase 1/3 (PC1/3) in the intestinal L-cells. Formation of glucagon is mediated by prohormone convertase 2 (PC2) in the pancreatic $\alpha$-cells. GRPP, glicentin-related pancreatic polypeptide; IP-1, intervening peptide-1; GLP-1, glucagon-like peptide-1. glucagon, rather than GLP-1, would be increased in individuals with systemic inflammation. To address this, we measured glucagon and GLP-1 in inflamed individuals using validated immunoassays $(16,17,19)$. In three prospective cohort studies including patients with (i) infective endocarditis (IE), (ii) urosepsis and iii) transcatheter aortic valve implantation/thoracic endovascular aortic repair (TAVI/TEVAR procedure), we correlated plasma concentrations of glucagon and GLP-1 to the well-known marker of inflammation, C-reactive protein (CRP). In a fourth study, we investigated the effect of acute systemic inflammation induced by an i.v. bolus infusion of bacterial lipopolysaccharide (LPS) on glucagon and GLP-1 concentrations in healthy men.

\section{Materials and methods}

\section{Study design and enrolment of patients}

Plasma samples were obtained from a prospective longitudinal cohort study conducted in the period from September 2015 to June 2017 at the Department of Urology, Department of Cardiology and Department of General Medicine at Herlev Hospital, Denmark, and at the Department of Cardiology at Rigshospitalet, Denmark. The primary endpoint and the study protocol have been described and published previously (20). A total of 49 hospitalized patients were divided into three cohorts based on the type of illness and presence of infection: (i) an infective endocarditis cohort $(n=16)$ comprising patients with newly diagnosed definite IE according to the modified DUKE criteria (mainly including bacteremia with Streptococcus spp., Enterococcus spp. or Staphylococcus aureus), (ii) a urosepsis cohort $(n=28)$ comprising patients with urinary tract bacteremia caused by Escherichia coli or inflammation: (iii) a TAVI/TEVAR (Transcatheter aortic valve implantation/thoracic endoVAscular repair) cohort $(n=5)$ comprising patients who underwent percutaneous aortic valve implantation or thoracic endovascular aortic repair and developed post-operative inflammation, but had no signs of infection. To meet the inclusion criteria, all patients were adults ( $\geq 18$ years), with a CRP concentration $\geq 30 \mathrm{mg} / \mathrm{L}$ and leucocytes $\geq 10 \times 10^{9} / \mathrm{L}$ at the time of inclusion. Patients with comorbidities such as rheumatic disease, cancer, disease- or medication-induced immunosuppression, patients with co-infections or surgery within 14 days prior to the study inclusion were excluded from the study population. All patients provided written informed consent. The study protocol was 
approved by the Data Protection Agency (HGH-2015-010, I-suite no: 03923) and the Danish Research Ethics Committee (H-15009681). All patients were treated during the hospitalization where the blood samples were collected, and had no relapse during 6 months of follow-up.

The experimental LPS study was conducted at Aarhus University Hospital, Skejby, Denmark, between December 2017 and October 2018. Nine healthy male participants without regular intake of medication, non-smokers, between 20 and 40 years of age, and BMI between 20 to $30 \mathrm{~kg} / \mathrm{m}^{2}$ were included. They received an i.v. bolus infusion of bacterial lipopolysaccharide (LPS, $1 \mathrm{ng} / \mathrm{kg}$ body weight) from E. coli (10,000 USP Endotoxin, Lot HOK354; the United States Pharmacopeia Convention, Inc., Rockville, Maryland) after a 10-h overnight fast. All participants gave written informed consent before participating in the study. The study protocol was approved by the Danish Research Ethics Committee (1-10-72-240-17) and registered on clinical trials (NCT03319550).

All studies were conducted in accordance with the Helsinki 2 declaration.

\section{Collection and storage of samples}

EDTA blood samples were collected from each patient and centrifuged at $3900 \boldsymbol{g}$ for $10 \mathrm{~min}$ (as described in (20)). The supernatant, plasma, was aliquoted, immediately frozen and kept at $-80^{\circ} \mathrm{C}$ until further analyses.

In the experimental LPS study, a total of $42 \mathrm{~mL}$ of blood was collected from each participant and centrifuged at $3600 \mathrm{~g}$ for $10 \mathrm{~min}$. The supernatant, plasma, was aliquoted, immediately frozen and kept at $-80^{\circ} \mathrm{C}$ until further analyses.

\section{Biochemical measurement of glucagon, GLP-1 and CRP}

Plasma concentration of glucagon was determined using a sandwich ELISA assay employing $\mathrm{N}$ - and C-terminus glucagon-specific antibody. This assay has previously been validated (19). Plasma concentration of total GLP-1 $\left(7-36 \mathrm{NH}_{2}+9-36 \mathrm{NH}_{2}\right)$ was determined by an in-house RIA specific for the amidated C-terminal (codename 89390). This assay measures not only intact GLP-1 but also its primary metabolite, GLP-1 9-36 $\mathrm{NH}_{2}$ (Fig. 1), which is formed almost immediately due to the actions of dipeptidylpeptidase-4 (DPP-4). Due to the rapid degradation, it is necessary to include the metabolite in the measurements to accurately estimate GLP-1 secretion (21). Measurements of CRP concentrations were performed using a COBAS®8000 modular analyzer series (Cobas-Roche).

In the LPS study, the acute systemic inflammatory response following LPS exposure was assessed by the measurement of TNF $\alpha$ and IL- 6 concentrations (Bio-Plex 48 Pro Human Chemokine Assay, Bio-Rad), and by changes in vital parameters (axillary temperature, heart rate, and mean arterial blood pressure $(\mathrm{MAP}=2 / 3 \times$ diastolic pressure $+1 / 3 \times$ systolic pressure)). The following symptoms: headache, shivering, nausea, back pain and muscle pain, were scored $(0=$ no symptoms, $5=$ worst symptoms ever experienced) and recorded every hour over 6-h period.

\section{Statistical analysis}

Distribution of data was assessed by histograms and qq plots. The degree of association between CRP and glucagon, and between CRP and GLP-1 was determined by nonparametric Spearman correlation analysis and simple linear regression. $T$-test was used to determine differences in glucagon, GLP-1 and CRP concentrations between fasting and non-fasting patients. In the experimental LPS study, a mixed effect model was used to determine the differences in TNF $\alpha$, IL-6, axillary temperature, heart rate, symptom score, mean arterial blood pressure, glucagon and GLP-1 concentrations between baseline (comparator) and the subsequent time points.

All statistical calculations were performed with GraphPad Prism (version 8.00, GraphPad Software, www. graphpad.com) and $P$-values $<0.05$ were considered significant for all analyses.

\section{Results}

\section{Study population}

Fourty-nine patients were enrolled in the prospective longitudinal clinical study. Twenty-seven females and 22 males with an average age of $69 \pm 13$ years (Table 1 ) established three cohorts based on the type of illness and presence of infection or inflammation.

Cohort 1 consisted of 16 patients (4 females, 12 males, average age of $66 \pm 13$ years) with bacterial left-sided infective endocarditis. Eighty-three plasma samples including 63 samples taken during fasting and 20 samples taken during non-fasting state were collected and analyzed.

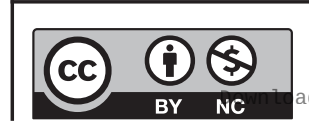

This work is licensed under a Creative Commons Attribution-NonCommercial 4.0 International License. ded from Bioscientifica.com at 04/26/2023 05:14:09AM via free access 
Table 1 Demographic and clinical characteristics of study patients.

\begin{tabular}{l}
\hline Variable \\
\hline Number of patients \\
Sex \\
$\quad$ Female \\
Male \\
Age, mean (S.D.) \\
Stratify age \\
$\quad \leq 49$ \\
$50-65$ \\
$65+$ \\
\hline
\end{tabular}

\begin{tabular}{c} 
All patients \\
\hline$n=49$ \\
$n(\%)$ \\
$27(55.1)$ \\
$22(44.9)$ \\
$69(13)$ \\
$n(\%)$ \\
$2(4.1)$ \\
$20(40.8)$ \\
$27(55.1)$ \\
\hline
\end{tabular}

\begin{tabular}{c}
\hline Cohort 1: infective endocarditis \\
\hline$n=16$ \\
$n(\%)$ \\
$4(25)$ \\
$12(75)$ \\
$66(13)$ \\
$n(\%)$ \\
$1(6.25)$ \\
$8(50)$ \\
$7(43.8)$
\end{tabular}

\begin{tabular}{c} 
Cohort 2: urosepsis \\
\hline$n=28$ \\
$n(\%)$ \\
$20(71.4)$ \\
$8(28.6)$ \\
$72(12)$ \\
$n(\%)$ \\
$1(3.6)$ \\
$10(35.7)$ \\
$17(60.7)$
\end{tabular}

\begin{tabular}{c}
\hline Cohort 3: TAVI/TEVAR \\
\hline$n=5$ \\
$n(\%)$ \\
$3(60.0)$ \\
$2(40.0)$ \\
$69(15)$ \\
$n(\%)$ \\
- \\
$2(40.0)$ \\
$3(60.0)$
\end{tabular}

P-value

Cohort 1 includes patients with infective endocarditis, cohort 2 includes patients with urosepsis, and cohort 3 includes patients who underwent percutaneous aortic valve implantation/replacement (TAVI/TEVAR, respectively). Nonparametric one-way ANOVA (Kruskal-Wallis) was used.

Cohort 2 consisted of 28 patients (20 females, 8 males, average age of $72 \pm 12$ years) with urosepsis. Seventy-one plasma samples including 53 samples taken during fasting and 18 samples taken during non-fasting states were collected and analyzed.

Cohort 3 consisted of five patients (three females, two males, average age of $69 \pm 15$ years) with non-infectious inflammation developed after TAVI/TEVAR procedure. Nineteen samples obtained during fasting were collected and analyzed.

\section{Glucagon, GLP-1 and CRP concentrations in the study population}

As expected, plasma concentrations of GLP-1 were significantly higher $(P<0.0001)$ in samples obtained during non-fasting states compared to samples obtained during fasting conditions, whereas no significant differences were observed for glucagon concentrations $(P=0.32)$ (Table 2). In addition to the fasting measurements, plasma concentrations of glucagon, GLP-1 and CRP were longitudinally assessed for each individual patient over the length of hospital stay (Fig. 2A, B and C).

\section{Correlation between CRP and glucagon and GLP-1}

A positive and significant correlation between CRP and glucagon was observed independently of fasting status (fasting + non-fasting patients: $\mathrm{r}=0.27, P=0.0003$; fasting patients: $r=0.28, P=0.0009$ ) (Fig. $3 \mathrm{~A}$ and $\mathrm{B}$, respectively). Subgroup correlation analyses between CRP and glucagon showed a positive correlation in cohort $1 \quad(\mathrm{r}=0.26$, $P=0.017$ ) (Fig. 3C), borderline significant correlation in cohort $2(\mathrm{r}=0.24, P=0.04)$ (Fig. 3D) and positive correlation in cohort $3(\mathrm{r}=0.63, P=0.004)$ (Fig. 3E).

In contrast, CRP and GLP-1 did not correlate significantly when merged datasets (fasting and non-fasting patients) and datasets of fasting patients only were analyzed $(\mathrm{r}=0.08, P=0.3$ and $\mathrm{r}=(-) 0.02$, $P=0.79$, respectively) (Fig. $4 \mathrm{~A}$ and $\mathrm{B}$, respectively). However, subgroup correlation analysis between CRP and GLP-1 revealed a positive correlation in cohort $2(\mathrm{r}=0.27$, $P=0.02$ ) (Fig. 4D).

\section{LPS study}

LPS infusion significantly increased TNF $\alpha$ and IL-6 plasma concentrations $(P=0.0001$ and $P=0.0027$, respectively), temperature $(P<0.0001)$, heart rate $(P<0.0001)$, symptom score $(P=0.0033)$, and decreased MAP $(P=0.014)$ (Fig. $5 \mathrm{~A}, \mathrm{~B}, \mathrm{C}, \mathrm{D}, \mathrm{E}$ and F). LPS administration also significantly increased plasma concentrations of glucagon (by $51 \pm 12 \% ; P=0.0065$ ) as compared to baseline (Fig. $6 \mathrm{~A}$ and B) but, in contrast, plasma GLP-1 concentrations were significantly decreased (by $55 \pm 5 \%$; $P=0.0098$ ) (Fig. 6A and C).

Table 2 Glucagon, GLP-1 and CRP levels in the study populations.

\begin{tabular}{|c|c|c|c|}
\hline Variable & All patients & $\begin{array}{c}\text { Fasted } \\
\text { patients }\end{array}$ & $\begin{array}{l}\text { Non-fasted } \\
\text { patients }\end{array}$ \\
\hline \multicolumn{4}{|l|}{ Glucagon (pmol/L) } \\
\hline Median (95\% Cl) & $5.0(4.4,5.6)$ & $5.0(4.3,5.9)$ & $5.3(4.0,9.1)$ \\
\hline Mean \pm S.E.M. & $8.1 \pm 0.8$ & $7.8 \pm 0.8$ & $9.4 \pm 2.1$ \\
\hline \multicolumn{4}{|l|}{ GLP-1 (pmol/L) } \\
\hline Median $(95 \% \mathrm{Cl})$ & $9(7,12)$ & $8(5,9)$ & $29(16,36)$ \\
\hline Mean \pm S.E.M. & $15 \pm 1$ & $11 \pm 1$ & $28 \pm 3^{* * *}$ \\
\hline \multicolumn{4}{|l|}{ CRP (mg/L) } \\
\hline Median $(95 \% \mathrm{Cl})$ & $44(35,72)$ & $39(24,73)$ & $56(31,124)$ \\
\hline Mean \pm S.E.M. & $80 \pm 6$ & $75 \pm 7$ & $96 \pm 15^{*}$ \\
\hline
\end{tabular}

Student's $t$-test was used to determine the differences between fasted and non-fasted patients.

*Reflects $P<0.05$ and *** Reflects $P<0.0001$.

$\mathrm{Cl}$, confidence intervals; CRP, c-reactive protein; GLP-1, glucagon-like peptide-1.

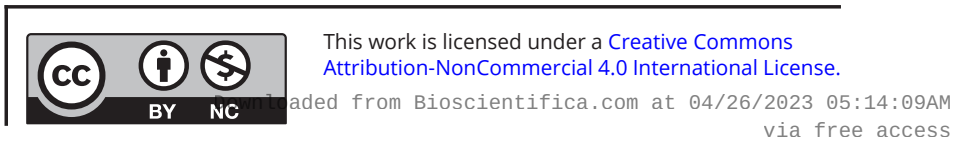



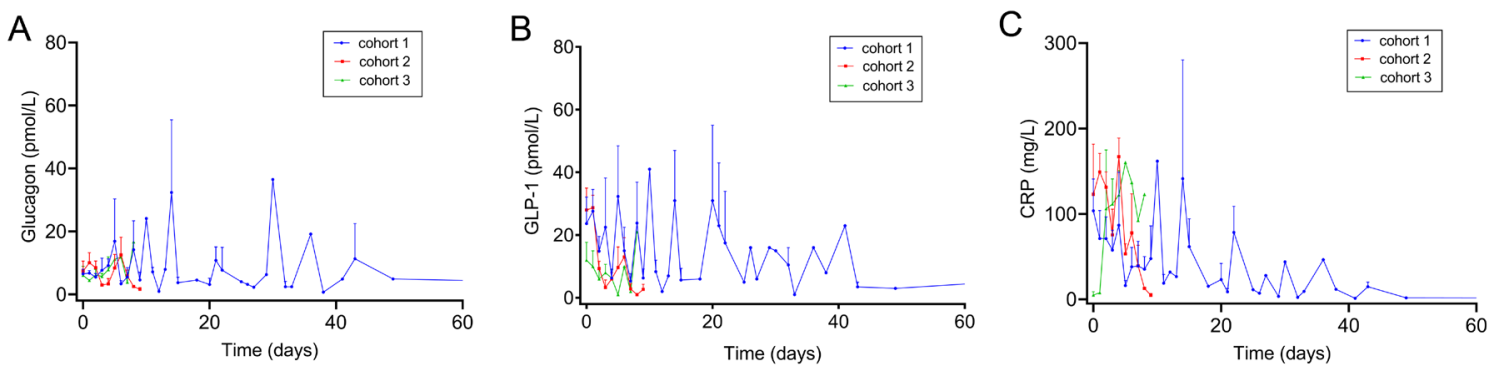

Figure 2

Changes in plasma concentrations of glucagon (A), glucagon-like peptide-1 (GLP-1) (B) and C-reactive protein (CRP) (C), over time in all patients (fasted and non-fasted) from the three cohorts. Two time points $(t=135$ and 145) in cohort 1 were omitted from the plots. Data are shown as mean + S.E.M.

\section{Discussion}

Here, we investigated plasma concentrations of glucagon and GLP-1 in patients with infection-induced inflammation (infective endocarditis and urosepsis cohorts: cohort 1 and 2) and inflammation due to percutaneous aortic valve implantation or thoracic endovascular aortic repair (TAVI/TEVAR cohort: cohort $3)$. We found a positive correlation between glucagon and CRP (a well-established biomarker used to assess systemic inflammation (22)) in the collective datasets including all patients, regardless of their fasting status, as well as in each of the three cohorts. In contrast, CRP and GLP-1 were not found to be correlated when collective datasets were analyzed. However, a subgroup analysis of urosepsis cohort (cohort 2) revealed a positive correlation between CRP and GLP-1. Administration of LPS increased glucagon concentrations by $50 \%$ and tended to decrease GLP-1 concentrations hours following the LPS bolus infusion.

Increased concentrations of glucagon and GLP-1 were recently reported in sepsis $(11,12)$ and critically ill patients (13), in mice following LPS injection (23), and in humans with burn lesions (24). Estimation of plasma GLP-1 in mice is troublesome due to the rapid degradation by neprilysin $(25,26)$, and the assays employed in the current human study and in other previously published studies also cross-react with GLP-1 $1-36 \mathrm{NH}_{2}$ that is co-secreted with glucagon (17). Therefore, one may speculate that the reported increases in GLP-1 actually reflect increased glucagon secretion from pancreatic alpha cells, as captured in the study presented here.

In the present work, significant correlation between GLP-1 and CRP was found only in cohort 2, including
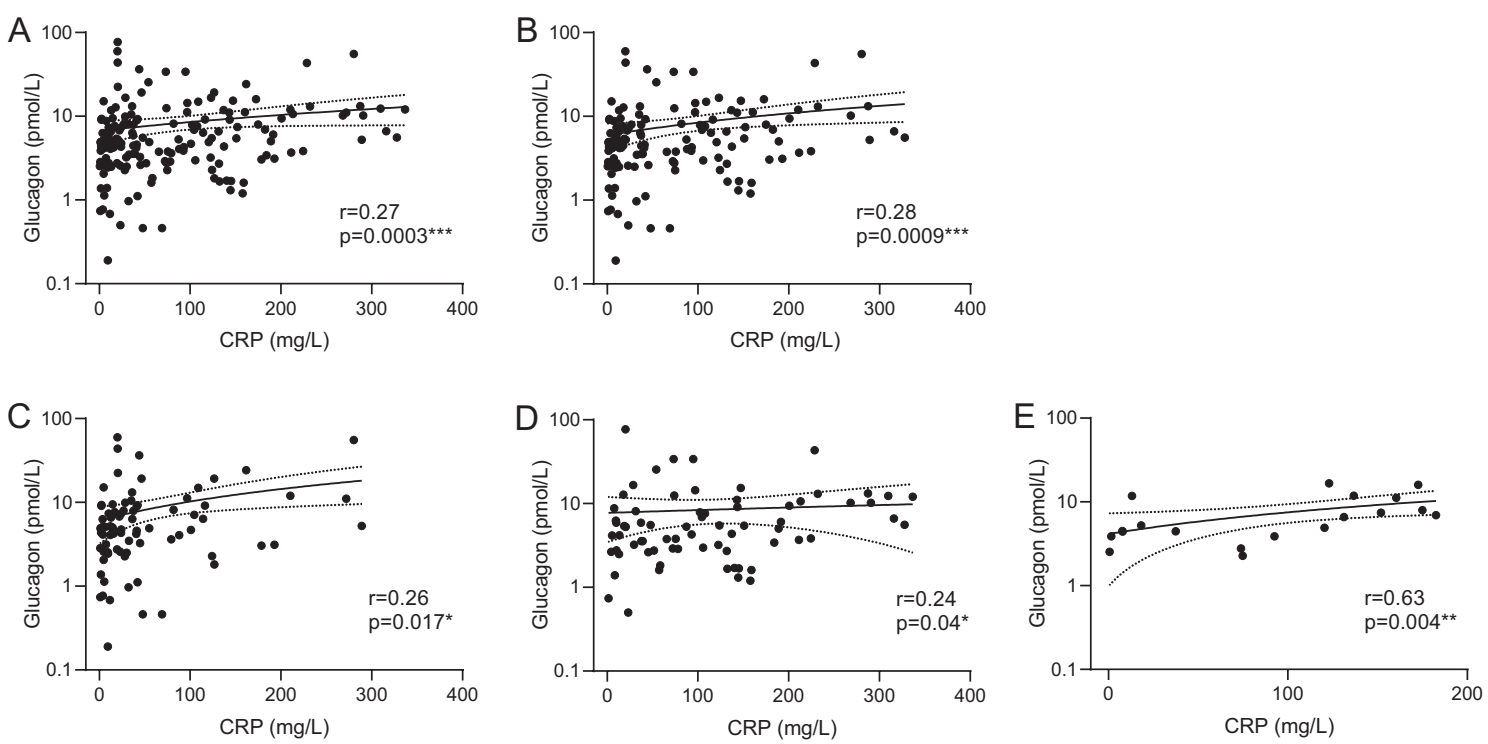

Figure 3

Correlation between C-reactive protein (CRP) and glucagon for all patients (A), fasted patients (B), all patients from cohort 1 (C), cohort 2 (D), cohort 3 (E). Glucagon variable is log scaled. Nonparametric Spearman correlation and simple linear regression were used.

https://ec.bioscientifica.com https://doi.org/10.1530/EC-20-0590 (c) 2021 The authors Published by Bioscientifica Ltd

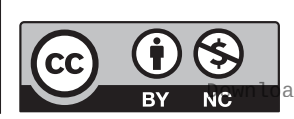

This work is licensed under a Creative Commons Attribution-NonCommercial 4.0 International License. ded from Bioscientifica.com at 04/26/2023 05:14:09AM via free access 

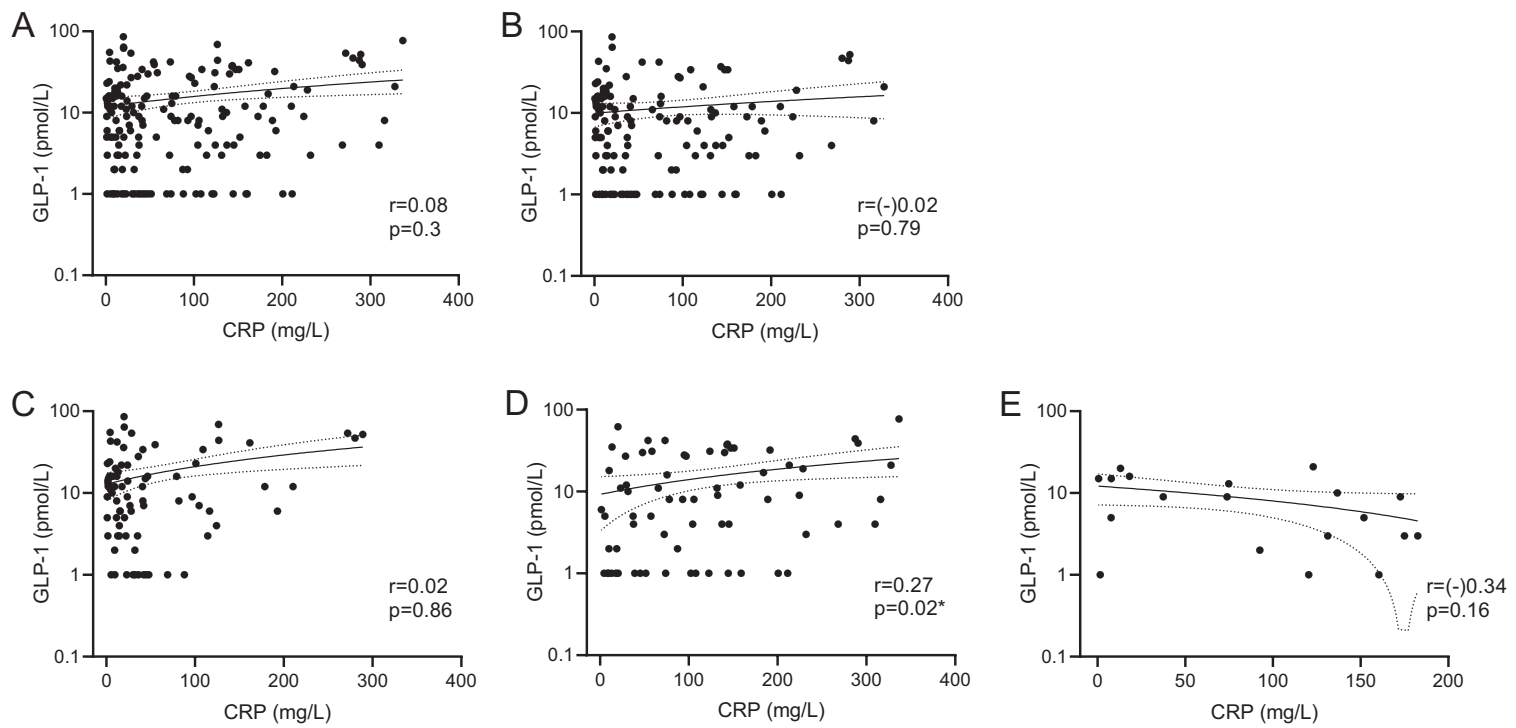

\section{Figure 4}

Correlation between C-reactive protein (CRP) and glucagon-like peptide-1 (GLP-1) for all patients (A), fasted patients (B), all patients from cohort 1 (C), cohort 2 (D), cohort 3 (E). GLP-1 variable is log scaled. Nonparametric Spearman correlation and simple linear regression were used.

patients with urosepsis (Fig. 5D). However, mean GLP-1 concentrations for all patients combined as well as for patients from each cohort separately were within the normal range (0-15 $\mathrm{pmol} / \mathrm{L})$.
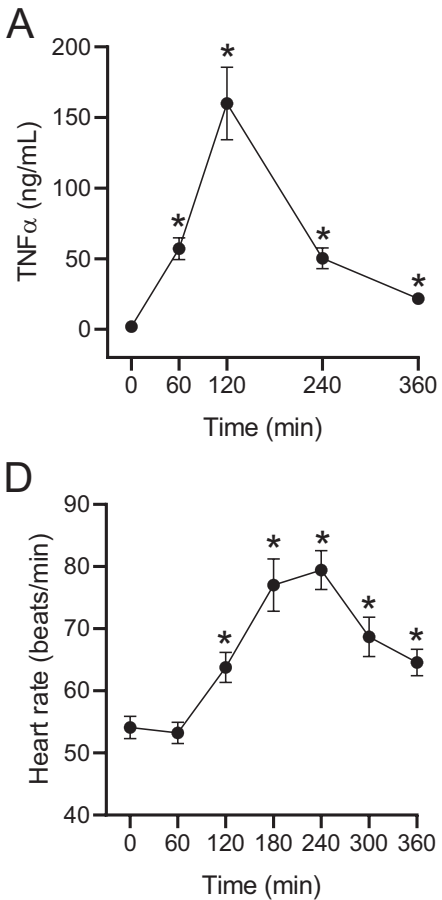

B

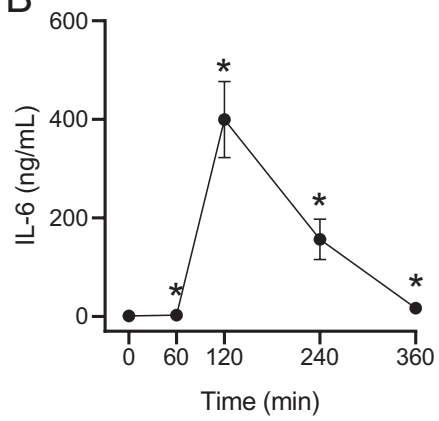

E

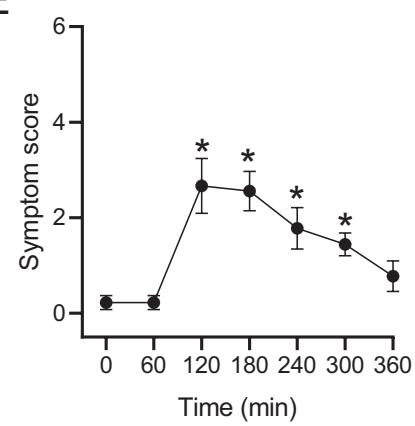

Our LPS infusion demonstrated that an acute induction of systemic inflammation was associated with 50\% increased glucagon but decreased GLP-1 concentrations. A lack of GLP-1 involvement in the course of infection was 
A

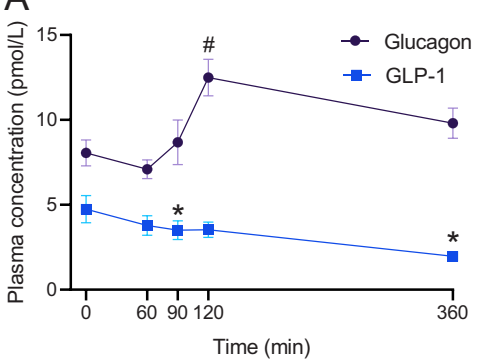

B

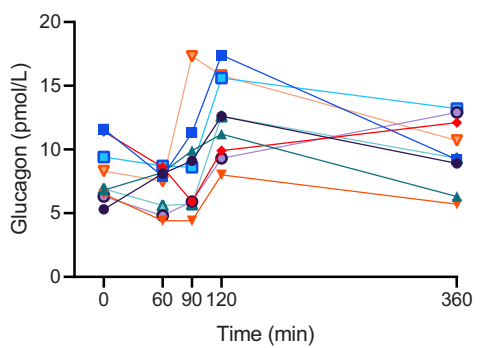

C

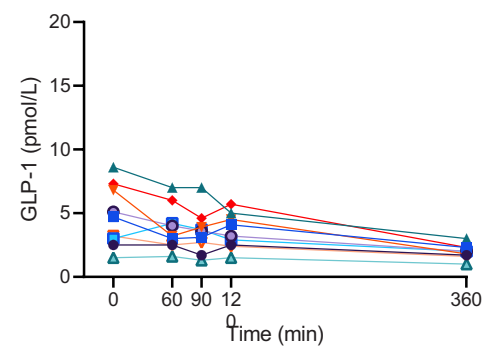

Figure 6

Changes in plasma concentrations of glucagon and glucagon-like peptide-1 (GLP-1) combined (A), glucagon (B) and GLP-1 (C) before ( $t=0$ ) and after $(\mathrm{t}=60,90,120,360)$ systemic lipopolysaccharide (LPS, $1 \mathrm{ng} / \mathrm{kg}$ body weight) exposure in young healthy males $(n=9)$. Each color represents individual patient (B and C). Data are shown as mean \pm S.E.M (C). A mixed effect model using baseline (time 0 ) as comparator was used to test statistical significance over time for glucagon and GLP-1 (* Reflects $P<0.05$ for GLP-1 and \# Reflects $P<0.05$ for glucagon).

also reported by Yin and co-workers (24). They showed no association between GLP-1 and white blood cell- (WBC), neutrophil- or lymphocyte counts.

Kidneys are the primary organ responsible for GLP-1 elimination (27), and elevated GLP-1 concentrations were found in patients with chronic kidney disease and renal failure (28). In our study significant correlation between GLP-1 and CRP was found exclusively in cohort 2 , including patients with urinary tract infection, which could be explained by the decreased kidney efficiency due to ongoing inflammation which results in delayed elimination of GLP-1. It is important to emphasize that it is not known whether or not a urinary tract infection affects renal elimination of GLP-1, and hence this explanation of increasing plasma levels remains speculative.

Elevated glucagon concentration was reported to be associated with disease severity and poorer clinical outcomes (multiple organs dysfunction and death) in severely ill patients with sepsis (13). As described previously, increased plasma concentrations of GLP-1 have been reported in conditions with sepsis; however, the exact mechanism(s) are not known. Increased glucagon and GLP-1 secretion might result from sympathicusdriven alpha cell activity giving rise to glucagon but also N-terminally elongated GLP-1 $\left(1-36 \mathrm{NH}_{2}\right)$, a proglucagon derived peptide that is co-secreted with glucagon from pancreatic alpha cells but can be detected by most total GLP-1 assays. Another possibility could be increased proteolysis in the case of severe sepsis that causes hyperaminoacidemia and consequently hyperglucagonemia (1).

In our study, glucagon concentrations were significantly correlated with CRP in all three cohorts as well as in the merged datasets, independent of fasting status. In addition, glucagon concentrations were significantly elevated in parallel with increased TNF $\alpha$, IL-6, heart rate, body temperature and symptoms of illness after systemic LPS exposure.

Due to the molecular heterogeneity of proglucagon derived molecules (Fig. 1), and because of the low (picomolar) concentrations of glucagon and GLP-1 in the systemic circulation, accurate measurements of both hormones are challenging in terms of accuracy and specificity $(16,17,29)$. Glucagon concentrations in response to hypoglycemia are typically ranging from 20 to $40 \mathrm{pmol} / \mathrm{L}$, but could decline down to $1-2 \mathrm{pmol} / \mathrm{L}$ in response to hyperglycemia. Therefore, only assays with high sensitivity $(0-1 \mathrm{pmol} / \mathrm{L})$ are suitable for the measurements of these low-abundant peptides. Based on our previous studies it is unlikely that we underestimated concentrations of glucagon or GLP-1 since the analytical methods employed in the present work are very sensitive and have been thoroughly validated $(16,17,19)$.

Many commercial kits available for glucagon and GLP-1 are far from being precise and accurate $(16,17)$, and the sensitivity and specificity may be different even between different batches (different LOT number) of the same kind. Moreover, protocols supplied by the manufacturer often miss essential information for example, about cross-reactions. Since the amino acid sequence of glucagon is also present in other peptides including oxyntomodulin or glicentin, and since the amino acid sequence of GLP- 1 shows approximately 50\% homology with glucagon, it is important to choose the most appropriate assays for a given project (18). Analytical methods based on antibodies' ability to recognize particular regions of the analyzed molecule require high specificity and sufficient binding energy. For example, antibodies against the mid-region of glucagon will also recognize oxyntomodulin and glicentin leading to crossreactions that compromise the specificity of the applied assay. Only simultaneous use of two different antibodies 
targeting both termini (i.e. sandwich ELISA) allows specific detection of glucagon molecule (19). In addition, interferences with highly abundant plasma components like albumin and immunoglobulin, may cause additional obstacles (18).

Another possible explanation for the results discrepancy between the cohorts could be the different origin of inflammation (i.e. bacterial infective endocarditis in cohort 1, bacterial urosepsis in cohort 2 and inflammation without signs of infection in cohort 3). Moreover, even though cohort 1 and 2 were characterized by bacterial infection, the nature and the course of the diseases are different. Urosepsis is a short-term infection, often with a rapid disease progression and relatively rapid recovery, whereas infective endocarditis is an infection where patients often have a long period of disease before diagnosis, and a long period of treatment and recovery. Each cohort could therefore has a distinct host-response pattern including different pattern of immune response and, eventually, distinct clinical outcomes.

\section{Strengths and limitations}

We exploited the strength of a longitudinal design where the individuals served as their own control. This approach enhances the power to identify statistically significant changes in plasma concentrations of glucagon, GLP-1 and CRP over time and helps to avoid potentially confounding inter-individual clinical variabilities. However, the observational nature of the study of the inflamed cohorts means that residual confounding cannot be excluded. However, the LPS study was an attempt to more directly evaluate the influence of acute inflammation on GLP-1 secretion. Another limitation is the relatively small sample size, and therefore additional investigations in larger cohorts are needed to further explore the association between GLP-1 and various inflammatory conditions. Our subgroup analysis of correlations between GLP-1 and CRP may therefore be underpowered.

\section{Conclusion}

In this study, we show that plasma glucagon concentrations correlated with CRP in patients with inflammation, whereas a similar correlation could not be identified for GLP-1. LPS infusion resulted in significantly increased glucagon, but not GLP-1 concentrations.
Inflammation and bacteremia may therefore be associated with increased glucagon, but not GLP-1 in humans. Increased plasma glucagon concentration (hyperglucagonemia) may potentially contribute to the hyperglycemia observed during inflammatory conditions, therefore antagonizing glucagon in such cases may be relevant (30).

\section{Declaration of interest}

The authors declare that there is no conflict of interest that could be perceived as prejudicing the impartiality of the research reported.

\section{Funding}

The study was supported by Rigshospitalet, University of Copenhagen, Denmark.

\section{Author contribution statement}

Nicolai J Wewer Albrechtsen and Jens J Holst designed, planned, interpreted and supervised the work. Christine F Klein, Henning Bundgaard, Maike Mose, Nikolaj Rittig and Niels Møller provided the clinical samples. Bolette Hartmann, Jens J Holst and Nicolai J Wewer Albrechtsen measured glucagon, GLP-1 and CRP. Justyna Modrzynska and Nicolai J Wewer Albrechtsen interpreted the results and wrote the manuscript. All authors revised and approved the final version of the manuscript.

\section{Acknowledgements}

The authors thank Christine Rasmussen from the Department of Clinical Biochemistry, Rigshospitalet, University of Copenhagen, and Lene Albæk from the Department of Biomedical Sciences, Faculty of Health and Medical Sciences, University of Copenhagen for the excellent laboratory and technical support. The authors wish to express their thanks to Jens Peter Gøtze (Department of Clinical Biochemistry, Rigshospitalet, University of Copenhagen) for helpful suggestions and fruitful discussions.

\section{References}

1 Wewer Albrechtsen NJ. Glucagon receptor signaling in metabolic diseases. Peptides 2018100 42-47. (https://doi.org/10.1016/j. peptides.2017.11.016)

2 Holst JJ, Albrechtsen NJW, Rosenkilde MM \& Deacon CF. Physiology of the incretin hormones, GIP and GLP-1-regulation of release and posttranslational modifications. Comprehensive Physiology 20199 1339-1381. (https://doi.org/10.1002/cphy.c180013)

3 Bell GI, Santerre RF \& Mullenbach GT. Hamster preproglucagon contains the sequence of glucagon and two related peptides. Nature 1983302 716-718. (https://doi.org/10.1038/302716a0)

4 Patzelt C \& Schiltz E. Conversion of proglucagon in pancreatic alpha cells: the major endproducts are glucagon and a single peptide, the major proglucagon fragment, that contains two glucagon-like sequences. PNAS 198481 5007-5011. (https://doi.org/10.1073/ pnas.81.16.5007)

5 Orskov C, Holst JJ, Poulsen SS \& Kirkegaard P. Pancreatic and intestinal processing of proglucagon in man. Diabetologia 198730 874-881. (https://doi.org/10.1007/BF00274797)

6 Müller TD, Finan B, Bloom SR, D'Alessio D, Drucker DJ, Flatt PR, Fritsche A, Gribble F, Grill HJ, Habener JF, et al. Glucagon-like peptide

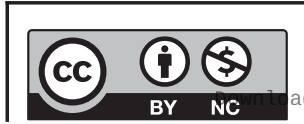


1 (GLP-1). Molecular Metabolism 201930 72-130. (https://doi. org/10.1016/j.molmet.2019.09.010)

7 Taeb AM, Hooper MH \& Marik PE. Sepsis: current definition, pathophysiology, diagnosis, and management. Nutrition in Clinical Practice 201732 296-308. (https://doi. org/10.1177/0884533617695243)

8 Gornik I, Vujaklija A, Lukić E, Madzarac G \& Gasparović V. Hyperglycemia in sepsis is a risk factor for development of type II diabetes. Journal of Critical Care 201025 263-269. (https://doi. org/10.1016/j.jcrc.2009.10.002)

9 Van Cromphaut SJ, Vanhorebeek I \& Van den Berghe G. Glucose metabolism and insulin resistance in sepsis. Current Pharmaceutical Design 200814 1887-1899. (https://doi. org/10.2174/138161208784980563)

10 Ali NA, O'Brien Jr JM, Dungan K, Phillips G, Marsh CB, Lemeshow S, Connors Jr AF \& Preiser JC. Glucose variability and mortality in patients with sepsis. Critical Care Medicine 200836 2316-2321. (https://doi.org/10.1097/CCM.0b013e3181810378)

11 Perl SH, Bloch O, Zelnic-Yuval D, Love I, Mendel-Cohen L, Flor H \& Rapoport MJ. Sepsis-induced activation of endogenous GLP-1 system is enhanced in type 2 diabetes. Diabetes/Metabolism Research and Reviews 201834 e2982. (https://doi.org/10.1002/dmrr.2982)

12 Brakenridge SC, Moore FA, Mercier NR, Cox M, Wu Q, Moldawer LL, Mohr AM, Efron PA \& Smith RS. Persistently elevated glucagon-like peptide-1 levels among critically ill surgical patients after sepsis and development of chronic critical illness and dismal long-term outcomes. Journal of the American College of Surgeons 201922958. e1-67.e1. (https://doi.org/10.1016/j.jamcollsurg.2019.04.014)

13 Jung WJ, Park BH, Chung KS, Kim SY, Kim EY, Jung JY, Kang YA, Kim YS, Kim SK, Chang J, et al. Glucagon levels, disease severity, and outcome in severe sepsis. Shock 201543 563-568. (https://doi org/10.1097/SHK.0000000000000344)

14 Kahles F, Meyer C, Möllmann J, Diebold S, Findeisen HM, Lebherz C, Trautwein C, Koch A, Tacke F, Marx N, et al. GLP-1 secretion is increased by inflammatory stimuli in an IL-6-dependent manner, leading to hyperinsulinemia and blood glucose lowering. Diabetes 201463 3221-3229. (https://doi.org/10.2337/db14-0100)

15 Lebherz C, Kahles F, Piotrowski K, Vogeser M, Foldenauer AC, Nassau K, Kilger E, Marx N, Parhofer KG \& Lehrke M. Interleukin-6 predicts inflammation-induced increase of Glucagon-like peptide-1 in humans in response to cardiac surgery with association to parameters of glucose metabolism. Cardiovascular Diabetology 2016 15 21. (https://doi.org/10.1186/s12933-016-0330-8)

16 Bak MJ, Albrechtsen NW, Pedersen J, Hartmann B, Christensen M, Vilsbøll T, Knop FK, Deacon CF, Dragsted LO \& Holst JJ. Specificity and sensitivity of commercially available assays for glucagon and oxyntomodulin measurement in humans. European Journal of Endocrinology 2014170 529-538. (https://doi.org/10.1530/EJE-13-0941)

17 Bak MJ, Wewer Albrechtsen NJ, Pedersen J, Knop FK, Vilsboll T, Jorgensen NB, Hartmann B, Deacon CF, Dragsted LO \& Holst JJ. Specificity and sensitivity of commercially available assays for glucagon-like peptide-1 (GLP-1): implications for GLP-1 measurements in clinical studies. Diabetes, Obesity and Metabolism 201416 1155-1164. (https://doi.org/10.1111/dom.12352)

18 Holst JJ \& Wewer Albrechtsen NJ. Methods and guidelines for measurement of glucagon in plasma. International Journal of Molecular Sciences 201920 5416. (https://doi.org/10.3390/ijms20215416)
19 Wewer Albrechtsen NJ, Hartmann B, Veedfald S, Windeløv JA, Plamboeck A, Bojsen-Møller KN, Idorn T, Feldt-Rasmussen B, Knop FK, Vilsbøll T, et al. Hyperglucagonaemia analysed by glucagon sandwich ELISA: nonspecific interference or truly elevated levels? Diabetologia 201457 1919-1926. (https://doi.org/10.1007/s00125014-3283-z)

20 Klein CF, Holle SLK, Andersen MH, Pedersen A, Bundgaard H, Iversen KK \& Malmendal A. In-hospital metabolite changes in infective endocarditis-a longitudinal (1). European Journal of Clinical Microbiology and Infectious Diseases 201938 1553-1560. (https://doi. org/10.1007/s10096-019-03586-z)

21 Orskov C, Rabenhoj L, Wettergren A, Kofod H \& Holst JJ. Tissue and plasma concentrations of amidated and glycine-extended glucagonlike peptide I in humans. Diabetes 199443 535-539. (https://doi. org/10.2337/diab.43.4.535)

22 Branco RG \& Garcia PC. Ferritin and C-reactive protein as markers of systemic inflammation in sepsis. Pediatric Critical Care Medicine 201718 194-196. (https://doi.org/10.1097/ PCC.0000000000001036)

23 Nguyen AT, Mandard S, Dray C, Deckert V, Valet P, Besnard P, Drucker DJ, Lagrost L \& Grober J. Lipopolysaccharides-mediated increase in glucose-stimulated insulin secretion: involvement of the GLP-1 pathway. Diabetes 201463 471-482. (https://doi.org/10.2337/ db13-0903)

24 Yin HN, Hao JW, Chen Q, Li F, Yin S, Zhou M, Zhang QH, Yao YM $\&$ Chai JK. Plasma glucagon-like peptide 1 was associated with hospital-acquired infections and long-term mortality in burn patients. Surgery 2020167 1016-1022. (https://doi.org/10.1016/j. surg.2020.01.004)

25 Windelov JA, Wewer Albrechtsen NJ, Kuhre RE, Jepsen SL, Hornburg D, Pedersen J, Jensen EP, Galsgaard KD, WintherSorensen M, Orgaard A, et al. Why is it so difficult to measure glucagon-like peptide-1 in a mouse? Diabetologia 201760 2066-2075. (https://doi.org/10.1007/s00125-017-4347-7)

26 Esser N, Barrow BM, Choung E, Shen NJ \& Zraika S. Neprilysin inhibition in mouse islets enhances insulin secretion in a GLP-1 receptor dependent manner. Islets 201810 175-180. (https://doi.org/ $10.1080 / 19382014.2018 .1502521)$

27 Orskov C, Andreasen J \& Holst JJ. All products of proglucagon are elevated in plasma from uremic patients. Journal of Clinical Endocrinology and Metabolism 199274 379-384. (https://doi. org/10.1210/jcem.74.2.1730817)

28 Meier JJ, Nauck MA, Kranz D, Holst JJ, Deacon CF, Gaeckler D, Schmidt WE \& Gallwitz B. Secretion, degradation, and elimination of glucagon-like peptide 1 and gastric inhibitory polypeptide in patients with chronic renal insufficiency and healthy control subjects. Diabetes 200453 654-662. (https://doi.org/10.2337/ diabetes.53.3.654)

29 Wewer Albrechtsen NJ, Veedfald S, Plamboeck A, Deacon CF, Hartmann B, Knop FK, Vilsboll T \& Holst JJ. Inability of some commercial assays to measure suppression of glucagon secretion. Journal of Diabetes Research 20162016 8352957. (https://doi. org/10.1155/2016/8352957)

30 Unger RH \& Cherrington AD. Glucagonocentric restructuring of diabetes: a pathophysiologic and therapeutic makeover. Journal of Clinical Investigation 2012122 4-12. (https://doi.org/10.1172/ JCI60016)

Received in final form 31 December 2020

Accepted 20 January 2021

Accepted Manuscript published online 23 January 2021 https://ec.bioscientifica.com https://doi.org/10.1530/EC-20-0590 (c) 2021 The authors Published by Bioscientifica Ltd

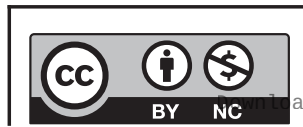

This work is licensed under a Creative Commons Attribution-NonCommercial 4.0 International License. ded from Bioscientifica com at 04/26/2023 05:14:09AM via free access 